\title{
Cooling and trapping of atomic strontium
}

\author{
Xinye Xu, Thomas H. Loftus, John L. Hall, Alan Gallagher, and Jun Ye \\ JILA, National Institute of Standards and Technology and University of Colorado, Boulder, Colorado 80309-0440
}

Received August 6, 2002; revised manuscript received December 5, 2002

\begin{abstract}
We present a detailed investigation of strontium magneto-optical trap (MOT) dynamics. Relevant physical quantities in the trap, such as temperature, atom number and density, and loss channels and lifetime, are explored with respect to various trap parameters. By studying the oscillatory response of a two-level ${ }^{1} S_{0^{-}}{ }^{1} P_{1}{ }^{88} \mathrm{Sr}$ MOT, we firmly establish the laser cooling dynamics predicted by Doppler theory. Measurements of the MOT temperature, however, deviate severely from Doppler theory predictions, implying significant additional heating mechanisms. To explore the feasibility of attaining quantum degenerate alkalineearth samples via evaporative cooling, we also present the first experimental demonstration of magnetically trapped metastable ${ }^{88} \mathrm{Sr}$. Furthermore, motivated by the goal of establishing the fermionic isotope ${ }^{87} \mathrm{Sr}$ as one of the highest-quality, neutral-atom-based optical frequency standards, we present a preliminary study of subDoppler cooling in a ${ }^{87} \mathrm{Sr}$ MOT. A dual-isotope $\left({ }^{87} \mathrm{Sr}\right.$ and $\left.{ }^{88} \mathrm{Sr}\right) \mathrm{MOT}$ is also demonstrated. (0) 2003 Optical Society of America
\end{abstract}

OCIS codes: $140.3320,020.7010,270.4180,300.1030,300.2530,300.6210$.

\section{INTRODUCTION}

Alkaline-earth metal atoms possess versatile internal level structure and a diversity of naturally abundant isotopes, making these systems extremely useful for fundamental studies of Doppler and recoil-limited laser cooling, ${ }^{1-20}$ precision optical-frequency metrology, ${ }^{21-25}$ and ultracold collisions. ${ }^{26-28}$ Specifically, the zeronuclear-spin isotopes are free from hyperfine structure and are thus ideal platforms for direct tests of Doppler cooling theory ${ }^{20}$ and quantitative studies of ultracold light-assisted collisions. In addition, the narrow intercombination transitions between singlet and triplet spin manifolds permit ultralow-temperature laser cooling ${ }^{12-18}$ in the interesting regime where the photon recoil energy exceeds the associated Doppler shift. Highly stable optical-frequency standards have also been demonstrated with the alkaline-earth intercombination lines. ${ }^{23,25}$ On the other hand, the nonzero-nuclear-spin isotopes, such as ${ }^{87} \mathrm{Sr}$, provide more attractive systems for optical frequency standards: The hyperfine structure of the ${ }^{1} S_{0}-{ }^{1} P_{1}$ transition may permit single-step sub-Doppler cooling to reach possibly tens of $\mu \mathrm{K}$ temperatures, while the ${ }^{1} S_{0}-{ }^{3} P_{0}$ intercombination line will provide a narrow clock transition that is highly insensitive to external perturbations. ${ }^{29}$ Moreover, the metastable ${ }^{3} P_{2}$ states present in both isotopes permit magnetic trapping ${ }^{30-32}$ and potentially evaporative cooling to Bose or Fermi degeneracy. Of the alkaline earths, atomic $\mathrm{Sr}$ is one of the most promising systems for these experiments.

This paper is organized as follows. In Section 2 we summarize our recent detailed study of oscillation dynamics in a ${ }^{88} \mathrm{Sr}$ magneto-optical trap (MOT). Here we find that although the trap spring constant and damping coefficient are well modeled by Doppler theory, the sample temperature greatly exceeds Doppler predictions. ${ }^{20}$ In addition we report optimum values of trapping-beam intensity and detuning that maximize trap density. Section 3 describes the unique attributes of ${ }^{87} \mathrm{Sr}$ that make this system ideal for a future highly accurate opticalfrequency standard and presents a preliminary study of sub-Doppler cooling in a ${ }^{87} \mathrm{Sr}$ MOT.

The desire to explore quantum degenerate gas-phase bosonic and fermionic mixtures has spawned increasing interest in dual-isotope and heteronuclear MOTs. ${ }^{33-36}$ In the case of atomic Sr, the high natural abundance of both bosonic $\left({ }^{88} \mathrm{Sr}, 82 \%\right.$ abundance) and fermionic $\left({ }^{87} \mathrm{Sr}, 7 \%\right.$ abundance) isotopes, coupled with the ability to cool individual isotopes with single-frequency lasers operating on the ${ }^{1} S_{0}{ }^{1} P_{1}$ transition, greatly simplifies the process of generating mixtures of bosons and fermions in a MOT. Moreover dual-isotope Sr MOTs offer the possibility of sympathetically cooling relatively hot ${ }^{88} \mathrm{Sr}$ atoms with sub-Doppler-cooled ${ }^{87} \mathrm{Sr}$ atoms. In Section 4 we describe the creation of a dual-isotope MOT for fermionic ${ }^{87} \mathrm{Sr}$ and bosonic ${ }^{88} \mathrm{Sr}$.

As a first step toward studies of photon-free cold collisions and radio-frequency evaporation of Sr, Section 5 describes our efforts to trap ${ }^{88} \mathrm{Sr}$ magnetically in the ${ }^{3} P_{2}$ metastable excited state. Currently we find that, although the trap population, loading rate, and density scale are as expected, blackbody radiation associated with the present vapor-cell trap limits the trap lifetime to $\sim 40$ ms. A beam-loaded apparatus operated at room temperature will extend the magnetic trap lifetime beyond several seconds.

\section{DOPPLER COOLING ${ }^{88}$ SR IN A MAGNETO-OPTICAL TRAP}

Figure 1 shows a simplified energy-level diagram for ${ }^{88} \mathrm{Sr}$. The ${ }^{1} S_{0}-{ }^{1} P_{1}$ transition $[\lambda=461 \mathrm{~nm}$, transition linewidth $(\mathrm{FWHM})=32 \mathrm{MHz}]$ is used to cool and trap ${ }^{88} \mathrm{Sr}$ in a standard six-beam MOT. In this blue ${ }^{88} \mathrm{Sr}$ MOT, atoms can leak out of the trap by radiatively branching from the ${ }^{1} P_{1}$ state to the metastable ${ }^{3} P_{2}$ state via the ${ }^{1} P_{1} \rightarrow{ }^{1} D_{2} \rightarrow{ }^{3} P_{2}$ decay path. Atoms decaying to the 


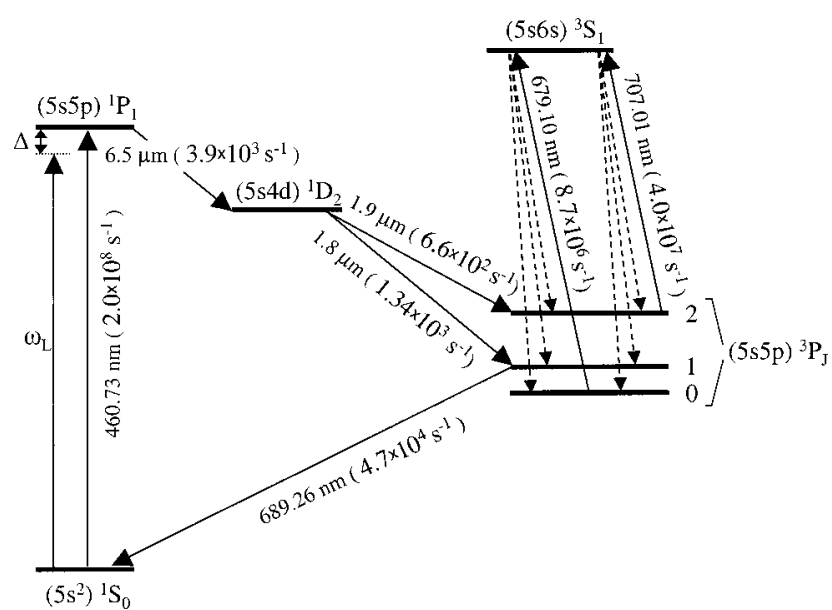

Fig. 1. Simplified energy level diagram for ${ }^{88} \mathrm{Sr}$ showing the main cooling transition at $461 \mathrm{~nm}$, radiative decay channels for the ${ }^{1} P_{1}$ excited state, and the relevant repumping scheme. Numbers in parentheses give the transition Einstein A coefficients. $\omega_{L}(\Delta)$ is the ${ }^{1} S_{0}-{ }^{1} P_{1}$ MOT cooling-laser frequency (detuning).

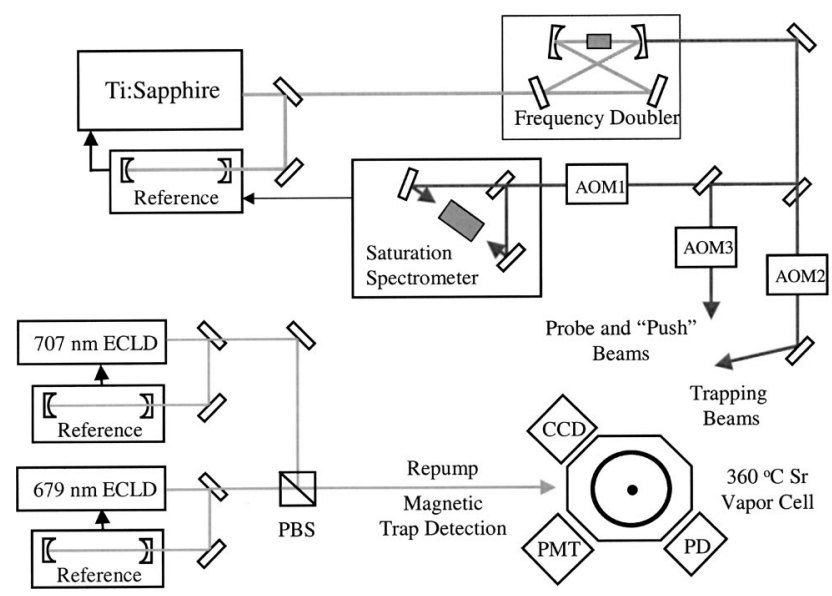

Fig. 2. ${ }^{1} S_{0}-{ }^{1} P_{1}$ vapor-cell Sr MOT. AOM, acousto-optic modulator; CCD, charge-coupled-device camera; PMT, photomultiplier tube; PD, photodiode; ECLD, external-cavity laser diode; PBS, polarizing beam splitter. Experimental results reported in this paper are obtained with appropriate modifications to this basic setup.

${ }^{3} P_{1}$ state are returned to the ground state, so the effective loss rate per ${ }^{1} P_{1}$ atom is $\sim 1.29 \times 10^{3} / \mathrm{s}$, which is quite small $(1: 160,000)$ in comparison with the strong cooling transition rate of $2.0 \times 10^{8} / \mathrm{s}$. Therefore ${ }^{8} \mathrm{Sr}$ MOTs can be produced simply by using single-frequency cooling light at $461 \mathrm{~nm}$. This capability is in contrast to all alkali-atom MOTs, in which repumping is an absolute necessity. To eliminate the ${ }^{3} P_{2}$ state shelving loss, two repumping lasers, one at $707 \mathrm{~nm}$ and the other at $679 \mathrm{~nm}$, are used to pump atoms from the ${ }^{3} P_{2}$ state to the ${ }^{3} S_{1}$ state. From the ${ }^{3} S_{1}$ state, the atoms decay to the shortlived ${ }^{3} P_{1}$ state, which then returns the atoms to the ground state. The $679-\mathrm{nm}$ laser is used to prevent repumping leaks to the ${ }^{3} P_{0}$ state. These repumping lasers increase the ${ }^{88} \mathrm{Sr}-\mathrm{MOT}$ density, the total number of atoms, and the trap lifetime typically by a factor of 10 . The lifetime is then limited by $\mathrm{Sr}-\mathrm{Sr}^{*}$ collisions, ${ }^{27}$ so this enhancement factor is intensity-dependent.
A simplified diagram of the Sr-vapor-cell, ${ }^{1} S_{0}-{ }^{1} P_{1}$ MOT is shown in Fig. 2. Various experimental results reported in this article were obtained with appropriate modifications to this basic setup. The 461-nm cooling and trapping light is generated by frequency-doubling the output from a Ti:sapphire laser. The cooling laser detuning is controlled by a double-pass acousto-optic modulator (AOM1). The intensity is stabilized by a second AOM (AOM2). A third AOM (AOM3) provides an on-resonance probe beam for absorption measurements. The two repumping beams are produced by external-cavity laser diodes (ECLD), each locked to a stabilized reference cavity. Since the cooling transition linewidths of the alkalineearth atoms are generally larger than those of the alkalimetal atoms, the magnetic quadrupole field gradient needs to be larger than the corresponding fields in alkalimetal MOTs. For the experiments reported here, we typically employ an axial field gradient $\left(\partial B_{z}\right)$ of $60 \mathrm{G} / \mathrm{cm}$.

In the absence of repumping lasers, the Sr-MOT loss rate $R_{L}$ is approximated by ${ }^{37,38}$

$$
R_{L}=R_{0}+\frac{I_{t} / 2 I_{s}}{1+I_{t} / I_{s}+4(\Delta / \Gamma)^{2}} A_{{ }_{P_{1} \rightarrow D_{2}}} B_{1_{D_{2} \rightarrow 3} P_{2}}
$$

where $R_{0}$ is the loss rate caused by background gas collisions and the second term on the right-hand side of Eq. (1) gives the loss rate that is due to radiative decay from the ${ }^{1} P_{1}$ state to the ${ }^{3} P_{2}$ state. $I_{t}$ is the total intensity of the six trapping beams, and $\Delta$ represents detuning. $I_{s}$ $=43 \mathrm{~mW} / \mathrm{cm}^{2}$ is the saturation intensity of the ${ }^{1} S_{0}$ $\rightarrow{ }^{1} P_{1}$ transition (such that $\Omega^{2}=\Gamma^{2} / 2, \Omega$ being the Rabi frequency), and $\Gamma / 2 \pi=32 \mathrm{MHz}$ is the transition linewidth. For $\mathrm{Sr}$ the radiative decay rate from the ${ }^{1} P_{1}$ state to the ${ }^{1} D_{2}$ state is $A_{1{ }_{1} \rightarrow} \rightarrow{ }^{1} D_{2}=3.85(1.47) \times 10^{3} / \mathrm{s},{ }^{39}$ and the predicted branching ratio from the ${ }^{1} D_{2}$ state to the ${ }^{3} P_{2}$ state is $B_{1} D_{2} \rightarrow{ }^{3} P_{2}=0.33 .{ }^{40}$ Figure 3 shows the measured ${ }^{88} \mathrm{Sr}$-MOT loss rate as a function of $I_{t}$ at a fixed $\Delta$. Here $\delta=\Delta / 2 \pi=-40 \mathrm{MHz}$, the axial magnetic field gradient $\partial B_{z}=53 \mathrm{G} / \mathrm{cm}$, and the Sr vapor pressure $p_{\mathrm{Sr}}$ is $3.5 \times 10^{-8}$ Torr (obtained at a cell temperature $T_{c}$ $=360^{\circ} \mathrm{C}$ ). To determine the loss rate, trap filling is observed by measuring absorption of an on-resonance, lowintensity probe beam that is focused through the trap center. A given loading curve is then fitted by a single exponential function. Finally the loss rate as a function

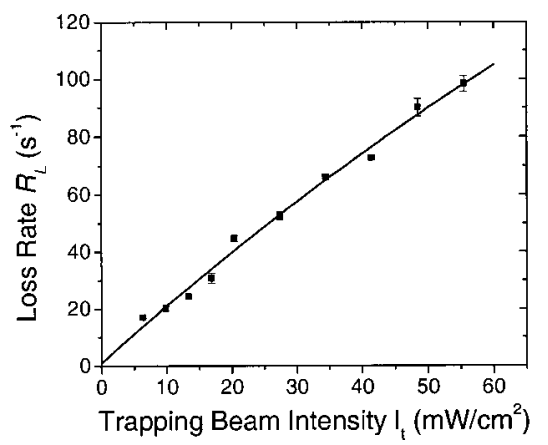

Fig. 3. Measured ${ }^{88}$ Sr MOT loss rate versus trapping-beam intensity for a fixed detuning of $-40 \mathrm{MHz}$. Filled squares are data and the curve is a least-squares fit according to Eq. (1). 
of the trapping laser intensity is fitted by $R_{L}=R_{0}$ $+\Gamma_{1} P_{1} \rightarrow{ }^{3} P_{2} I_{t} /\left(624+2 I_{t}\right)$ in accordance with Eq. (1), where $\Gamma^{1} P_{1} \rightarrow{ }^{3} P_{2}=A_{1 P_{1} \rightarrow{ }^{1} D_{2}} B^{1} D_{D_{2} \rightarrow{ }^{3} P_{2}}$. We find that the fitted value of $\Gamma_{1 P_{1} \rightarrow{ }^{3} P_{2}}=1.29(0.04) \times 10^{3} \mathrm{~s}^{-1}$ is in excellent agreement with the predicted rate $\Gamma_{1} P_{1} \rightarrow{ }^{3} P_{2}$ $=1.29(0.26) \times 10^{3} \mathrm{~s}^{-1}$. According to the fit in Fig. $3, R_{0}$ is less than $1 \mathrm{~s}^{-1}$. In the absence of repumping, the ${ }^{88} \mathrm{Sr}$-MOT lifetime is therefore dominated by decay to the metastable dark state ${ }^{3} P_{2}$, and thus can be expressed as

$$
\tau=1.56(0.05) \times 10^{-3}\left[1+\frac{1+4(\Delta / \Gamma)^{2}}{I_{t} / I_{s}}\right] \mathrm{s} .
$$

This result is in contrast to ordinary alkali-metal MOTs, where the lifetime is typically determined by the loss from background collisions.

On the basis of the loading curves and measured trap sizes, we can calculate the peak density $n_{0}$ from $n_{0}$ $=-1 /\left(\sigma r_{e} \sqrt{\pi}\right) \ln \left(I / I_{0}\right)$, where $\sigma=3 \lambda^{2} / 2 \pi$ is the absorption cross section, $r_{e}$ is the $1 / e$ atomic cloud radius, and $I$ $\left(I_{0}\right)$ is the probe-beam transmitted intensity when the MOT is switched on (off). The cloud density distribution is described by $n=n_{0} \exp \left(-r^{2} / r_{e}^{2}\right)$. Figure 4(a) shows $n_{0}$ versus the trapping-beam intensity for $\delta=-40 \mathrm{MHz}$, $\partial B_{z}=53 \mathrm{G} / \mathrm{cm}$, and $p_{\mathrm{Sr}}=3.5 \times 10^{-8}$ Torr under the condition of no repumping lasers. Under these conditions, the intensity that optimizes $n_{0}$ is $\sim 20 \mathrm{~mW} / \mathrm{cm}^{2}$. For intensities below (above) this value, $n_{0}$ decreases (plateaus). These results can be understood in terms of the competition between the trap loss rate (as shown in Fig. 3) and the trapping-beam capture velocity $v_{c}$. Specifically, as the trapping-beam intensity increases, both $R_{L}$ and $v_{c}$ increase while the cloud size remains essentially constant, leading to a peak density that is weakly dependent on the intensity above a critical value. This argument can be solidified with a simple theory. The solid curve in Fig. 4(a) shows a theoretical prediction based on $n_{0}=L /\left(R_{L} \pi^{3 / 2} r_{e}{ }^{3}\right)$, where $L=0.5 n_{\mathrm{Sr}} V^{2 / 3}$ $\times v_{c}{ }^{4}\left(m_{\mathrm{Sr}} / 2 k_{B} T_{c}\right)^{3 / 2}$ is the loading rate, ${ }^{8} T_{c}$ is the vapor cell temperature, $n_{\mathrm{Sr}}$ is the $\mathrm{Sr}$ background vapor density, $m_{\mathrm{Sr}}$ is the $\mathrm{Sr}$ mass, and $V=4 \pi R_{e}{ }^{3} / 3$ is the capture volume, with $R_{e}$ being the $1 / e$ trapping-beam radius $(\sim 5$ $\mathrm{mm}) . \quad v_{c}$ is obtained by numerically modeling the onedimensional capture process for $\mathrm{Sr}$ atoms. ${ }^{9}$ Figure 4(b) shows the trap peak density as a function of the trappinglaser detuning for $I_{t}=40 \mathrm{~mW} / \mathrm{cm}^{2}, \partial B_{z}=62 \mathrm{G} / \mathrm{cm}$, and $p_{\text {Sr }}=3.5 \times 10^{-8}$ Torr, in the presence (data in filled circles) and absence (data in filled squares) of repumping lasers. Solid curves in the figure show theoretical predictions based on the model described above. Clearly there is an optimal detuning, $\sim 45 \mathrm{MHz}$, for the maximum trap density. As the detuning increases, the loss rate decreases while the capture velocity first grows and then decreases, as deceleration of atoms in the trapping region becomes less efficient. We find the optimal detuning of about $45 \mathrm{MHz}$ is the same with or without repumping lasers. However, the density is increased by a factor of 10 when the repumping lasers are present. In Fig. 4 the calculated results based on the simplistic onedimensional model agree only qualitatively with the measured trap densities. The model, however, does reproduce the essential trends observed in the data.
Motivated by the data shown in Fig. 3, we optimized the operation of the ${ }^{88} \mathrm{Sr}$ MOT by adding appropriate repumping lasers. Figure 5 illustrates improvements in the trap lifetime and the atom number, both by a factor of 10 , as a result of the two repumping lasers employed. The data were obtained by using trap fluorescence to measure trap loading curves under the following conditions: $I_{t}=27 \mathrm{~mW} / \mathrm{cm}^{2}, \delta=-46 \mathrm{MHz}, \partial B_{z}=44 \mathrm{G} / \mathrm{cm}$, and $p_{\mathrm{Sr}}=2.1 \times 10^{-8}$ Torr.

Given the atomic two-level-like structure, the ${ }^{88} \mathrm{Sr}$ ${ }^{1} S_{0}-{ }^{1} P_{1}$ MOT provides an ideal system for testing Doppler cooling theory. By monitoring the absorption signal of a focused probe beam positioned a half radius from the center of the trap, we measured the step response of center-of-mass trap oscillations produced by a chopped
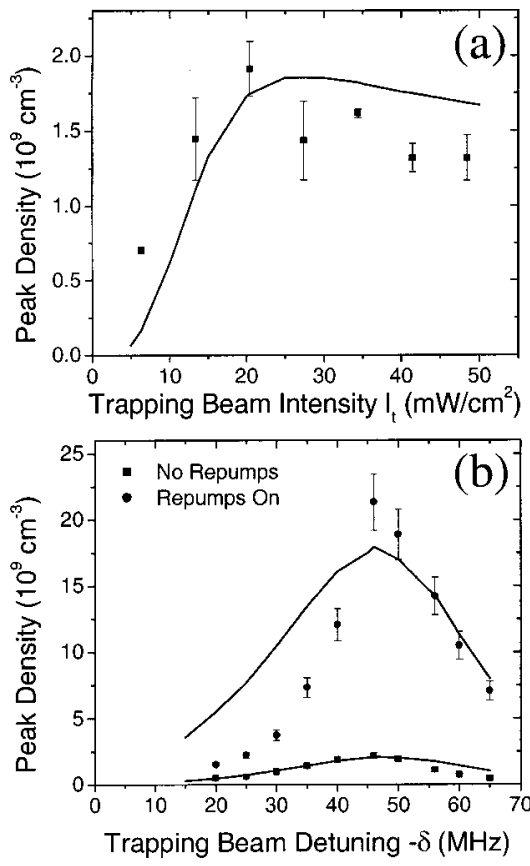

Fig. 4. Dependence of the ${ }^{88} \mathrm{Sr}$ MOT density on (a) trappingbeam intensity and (b) trapping-beam detuning. Theoretical predictions are shown as the solid curves.

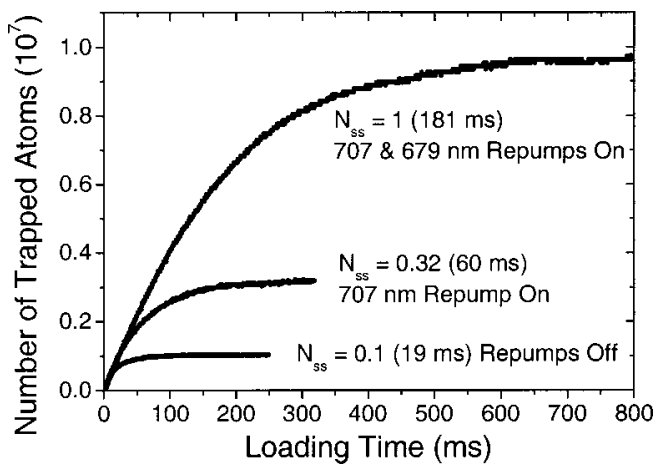

Fig. 5. Effect of the repumping lasers on the ${ }^{88} \mathrm{Sr}$ MOT population and lifetime for $I_{t}=27 \mathrm{~mW} / \mathrm{cm}^{2}, \delta=-46 \mathrm{MHz}$, and $\partial B_{z}$ $=44 \mathrm{G} / \mathrm{cm}$. $\quad N_{\mathrm{ss}}$ is the steady-state MOT population $\left(\times 10^{-7}\right)$. The numbers in parentheses are the corresponding trap lifetimes. 


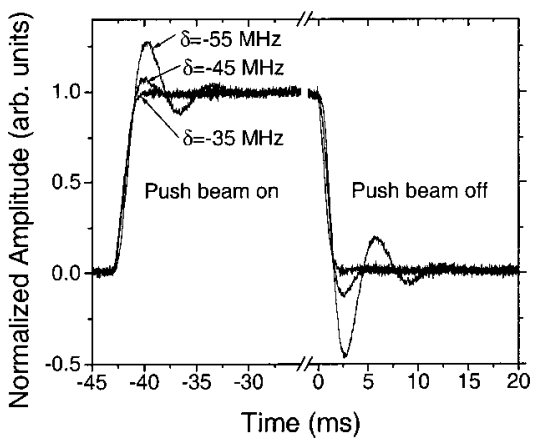

Fig. 6. Damped ${ }^{88}$ Sr MOT oscillation signals during a pushingbeam chopping cycle for three different detunings. The intensity of the trapping beam is $27 \mathrm{~mW} / \mathrm{cm}^{2}$ and $\partial B / \partial z=53 \mathrm{G} / \mathrm{cm}$.
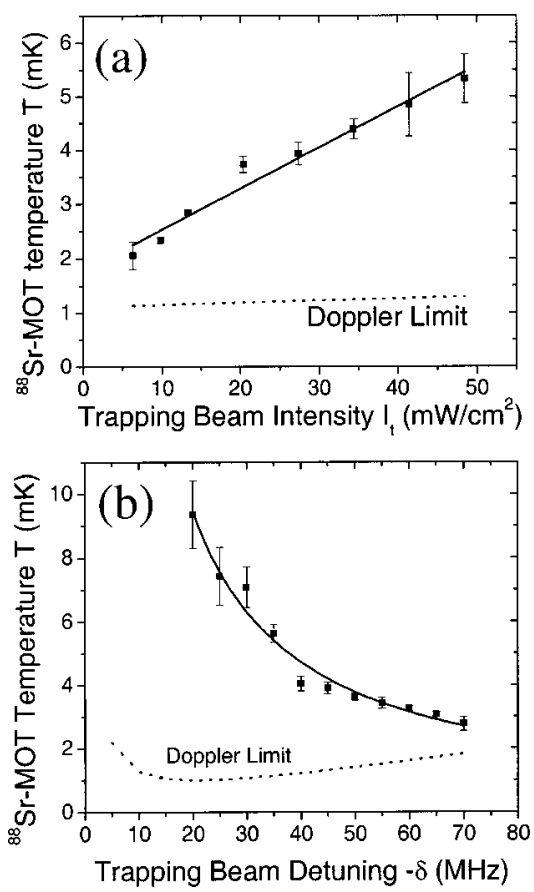

Fig. 7. Dependence of the trap temperature on (a) the trappingbeam intensity and (b) detuning as determined from the measured $\kappa$ values and trap sizes. Fits are shown by solid curves. Predictions based on Doppler theory are shown as dashed curves. In (a) the measured slope is $0.076(0.005)$ while the theoretical slope is $0.0036(0.0007) \mathrm{mK} /\left(\mathrm{mWcm}^{-2}\right)$.

push beam aligned perpendicular to the probe beam in the horizontal plane. ${ }^{20}$ Figure 6 shows probe transmission signals during one on-off cycle without repumping lasers and for several trapping laser detunings. Here $I_{t}$ $=27 \mathrm{~mW} / \mathrm{cm}^{2}, \quad \partial B_{z}=53 \mathrm{G} / \mathrm{cm}, \quad$ and $\quad p_{\mathrm{Sr}}=3.5$ $\times 10^{-8}$ Torr. When the push beam is on (off), the cloud center-of-mass is displaced (returns to its original position). In both cases the cloud exhibits damped harmonic oscillation as it approaches equilibrium. We can express the spring constant as $\kappa=4 \pi^{2} m_{\text {Sr }} f$ and the damping coefficient $\alpha=4 \pi m_{\mathrm{Sr}} \xi f$, where $f$ is the oscillation frequency and $\xi$ is the dimensionless quantity that represents the damping strength. Clearly, as shown in Fig. 6, the oscillation frequency decreases and the oscillation amplitude increases when the trapping laser detuning increases, implying that both the spring constant and the damping coefficient are inversely proportional to the de- tuning. Since there are no extra optical fields in the freeoscillation period when the push beam is turned off, these oscillation signals can be used to deduce the trap spring constant and damping coefficient. ${ }^{20}$ By fitting the data to our model, we find that the measured damping coefficients and spring constants are consistent with Doppler theory predictions at the level of $10 \%$. Based on the equipartition theorem, $(1 / 2) k_{B} T=1 / 2 \kappa r_{\mathrm{rms}}^{2}$, the cloud temperature $T$ can be determined from $\kappa$ and the trap size. These results are shown in Fig. 7 for $\delta$ $=-40 \mathrm{MHz}, \quad p_{\mathrm{Sr}}=3.5 \times 10^{-8}$ Torr, and $\partial B_{z}$ ranging from $35 \mathrm{G} / \mathrm{cm}$ to $80 \mathrm{G} / \mathrm{cm}$. Temperatures obtained with this procedure were independently verified at the level of $10 \%$ with a cloud-expansion technique. Additionally we find that for a given intensity, the measured temperature is independent of $\partial B_{z}$. For comparison the Doppler cooling limit is also displayed in the figure. The Doppler limit is expressed as ${ }^{1-5,24,41}$

$$
T_{D}=\frac{\hbar \Gamma}{8 k_{B}} \frac{1+I_{t} / I_{s}+4(\Delta / \Gamma)^{2}}{|\Delta| / T} .
$$

On the basis of this expression, the data shown in Fig. 7(a) are fitted by $T=T_{0}+a I_{t}$. From the fit we find $T_{0}=1.77(0.15) \mathrm{mK}, \quad a=0.076(0.005)\left(\mathrm{mK} \mathrm{cm}^{2}\right) /$ $\mathrm{mW}$; the corresponding theoretical values are $T_{0}$ $=1.11(0.22) \mathrm{mK}$ and $a=0.0036(0.0007)\left(\mathrm{mK} \mathrm{cm}^{2}\right) /$ $\mathrm{mW}$. Therefore, while the measured temperature is close to the predicted values in the low-intensity limit, it rises much faster with intensity than Doppler theory predicts, by more than a factor of 20. As shown in Fig. 7(b), we find that as $|\Delta|$ decreases the measured temperature increases rapidly compared to the Doppler predictions, especially in the region below $40 \mathrm{MHz}$. Here $I_{t}$ $=27 \mathrm{~mW} / \mathrm{cm}^{2}$ and $\partial B_{z}=53 \mathrm{G} / \mathrm{cm}$. By analogy to Eq. (2), we fit the data to $T=c|\delta|+d /|\delta|$, where $c$ and $d$ are two fitting parameters. From the fit, $c=-0.00014$ (0.00364) $\mathrm{mK} / \mathrm{MHz}$ and $d=189$ (6) $\mathrm{mK} \mathrm{MHz}$; in essence $T \propto 1 /|\delta|$. The corresponding theoretical values are $c$ $=0.0239(0.0048) \mathrm{mK} / \mathrm{MHz}$ and $d=10(2) \mathrm{mKMHz}$. Thus the measured intensity and detuning dependence both deviate severely from Doppler theory. Combining these observations of trap dynamics and temperature, we conclude that the cooling rate (represented by the damping coefficient) predicted by Doppler cooling theory agrees well with experimental results, but the heating rate does not. We are currently investigating possible causes for the extra heating effects.

\section{SUB-DOPPLER COOLING ${ }^{87}$ SR IN A MAGNETO-OPTICAL TRAP}

To date, highly stable optical-frequency standards have been demonstrated by using the intercombination lines in even-isotope alkaline earth. ${ }^{23,25}$ In order to reach $\mu \mathrm{K}$ temperatures, two-stage Doppler cooling was essential; thus the system becomes rather complicated. Additionally, the corresponding optical transition linewidths are always above the hertz level and are sensitive to cold- 


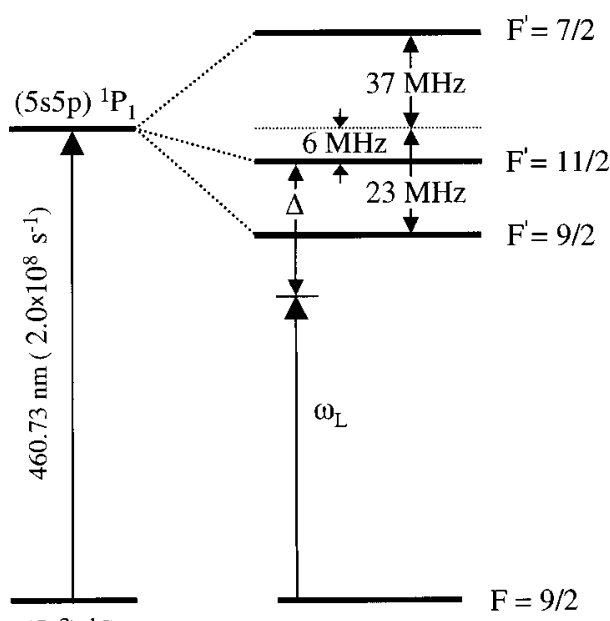

$\left(5 s^{2}\right){ }^{1} S_{0}$

Fig. 8. Hyperfine structure of the ${ }^{1} S_{0}{ }^{1} P_{1}$ fermionic ${ }^{87} \mathrm{Sr}$ cooling and trapping transition. $\omega_{L}(\Delta)$ is the ${ }^{1} S_{0}-{ }^{1} P_{1}$ MOT cooling-laser frequency (detuning). The ${ }^{1} S_{0}-{ }^{1} P_{1}$ Einstein A coefficient is given in parentheses.

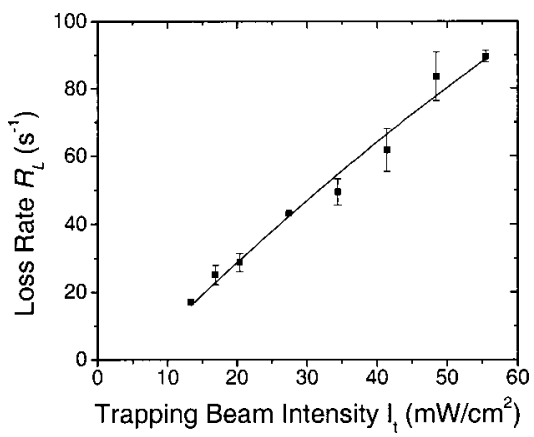

Fig. 9. Measured ${ }^{87} \mathrm{Sr}$ MOT loss rate versus trapping-beam intensity for $\delta=-40 \mathrm{MHz}$. The solid curve is a least-squares fit according to Eq. (1).

collision shifts. However in the fermionic alkaline-earth ${ }^{87} \mathrm{Sr}$, there is an extremely stable and narrow optical transition, ${ }^{1} S_{0}(F=9 / 2)-{ }^{3} P_{0}\left(F^{\prime}=9 / 2\right)$, with a linewidth of $\sim 1 \mathrm{mHz}$, which is far less sensitive to external electromagnetic fields and cold-collision shifts. (Note that the collision shift is usually scaled by the relevant transition linewidth.) When considering a Lamb-Dicke regime for these neutral atoms using a magic wavelength ${ }^{14}$ for a far-off-resonance lattice trap, the insensitivity to the light polarization of the $J=0$ states will be invaluable in practice and important for clock accuracy. Furthermore the temperature of ${ }^{87} \mathrm{Sr}$ may be reduced to tens of microkelvins by use of a single-stage, polarization-gradient cooling in a ${ }^{1} S_{0^{-}}{ }^{1} P_{1}$ MOT.

Motivated by these system advantages, we have cooled and trapped fermionic ${ }^{87} \mathrm{Sr}$ by slightly modifying the ${ }^{88} \mathrm{Sr}$ MOT setup shown in Fig. 2. The atomic energy levels relevant to cooling and trapping of ${ }^{87} \mathrm{Sr}$ are shown in Fig. 8. Since there are magnetic sublevels in the ground ${ }^{1} S_{0}$ state, sub-Doppler cooling is operative in a ${ }^{87} \mathrm{Sr}$ MOT. On the other hand, the Landé g-factor for the ground state is much smaller than those in alkali-metal atoms, leading to more efficient sub-Doppler cooling in the presence of applied magnetic fields. ${ }^{6-11}$ Figure 9 demon- strates that the ${ }^{87} \mathrm{Sr}$ MOT loss rate is also primarily determined by radiative branching from the ${ }^{1} P_{1}$ excited state. Here the conditions are $\delta=-40 \mathrm{MHz}, \partial B_{z}$ $=53 \mathrm{G} / \mathrm{cm}$, and $p_{\mathrm{Sr}}=5.6 \times 10^{-8}$ Torr $\left(T_{c}=370^{\circ} \mathrm{C}\right)$. Fitting Eq. (1) to the observed loss rates, we find $\Gamma^{{ }^{1} P_{1} \rightarrow{ }^{3} P_{2}}=1.32(0.07) \times 10^{3} \mathrm{~s}^{-1}$, in good agreement with the theoretically predicted value. However, for ${ }^{87} \mathrm{Sr}$, a more elaborate scheme of repumping lasers (for example, using frequency modulation to broaden the repump bandwidth) must be employed to achieve efficient recycling. Figure 10(a) shows the measured ${ }^{87} \mathrm{Sr}$ MOT temperature as a function of the trapping beam intensity for a fixed laser detuning of $\delta=-40 \mathrm{MHz}$. Figure 10(b) shows the measured temperature as a function of the laser detuning for fixed trapping beam intensities of $I_{t}=48$ and 27 $\mathrm{mW} / \mathrm{cm}^{2}$. In all cases, the magnetic field gradient is $\partial B_{z}=53 \mathrm{G} / \mathrm{cm}$ and $p_{\mathrm{Sr}}=5.6 \times 10^{-8}$ Torr. For comparison, dashed curves show the temperature dependence expected from Doppler theory. In both plots, sub-Doppler cooling is clearly revealed.

To perform these temperature measurements, we use a cloud expansion technique., ${ }^{7,20}$ A 100 - $\mu$ m-diameter, onresonance, 461-nm probe beam is sent through the center of the trap, and absorption of the probe beam is detected by a photodiode. Trap decay curves are recorded after the MOT is switched off. The data are then fitted with

$$
f(t)=\zeta_{1} \exp \left(-\frac{\zeta_{2}}{r_{0}{ }^{2}+2 v_{\mathrm{rms}}{ }^{2} t^{2}}\right),
$$

where $\zeta_{1}, \zeta_{2}$, and $v_{\mathrm{rms}}$ are three fitting parameters and $r_{0}=\left(r_{e x} r_{e y} r_{e z}\right)^{1 / 3}$, where $r_{e x}, r_{e y}$, and $r_{e z}$ are the $1 / e$ radii of the cloud as determined from fits to images of the 461-nm trap fluorescence. From the fitting param-
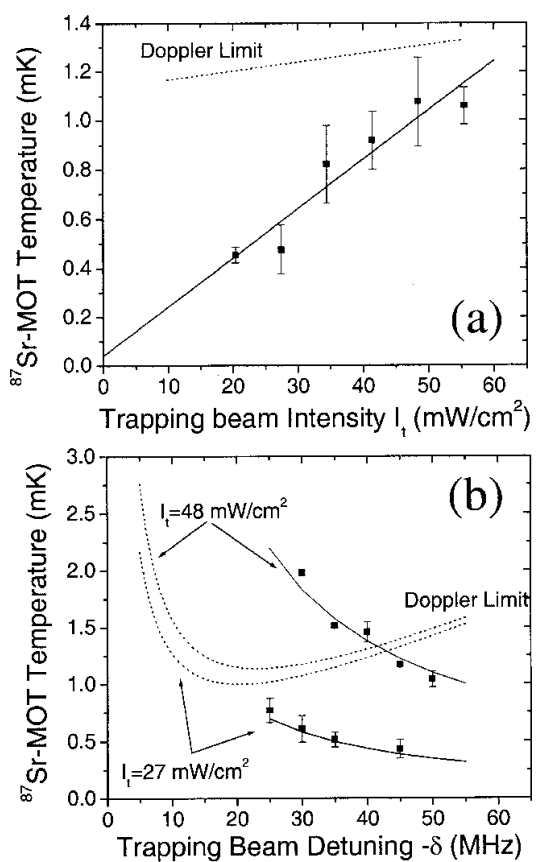

Fig. 10. Sub-Doppler cooling of ${ }^{87} \mathrm{Sr}$ in a ${ }^{1} S_{0^{-}}{ }^{1} P_{1}$ MOT. The dependence of the trap temperature is shown with respect to (a) trapping beam intensity and (b) detuning. For comparison, the Doppler cooling limit for each case is displayed as a dashed curve. 
eter $v_{\text {rms }}$ we obtain the trap temperature from $T$ $=m v_{\mathrm{rms}}^{2} / k_{B}$. In Fig. 10, according to the sub-Doppler cooling theory, ${ }^{6-11}$ we fit the data by $T=a(\hbar \Gamma /$ $\left.2 k_{B}\right)\left(I_{t} / I_{s}\right) /(|\Delta| / \Gamma)+b$, where $a$ and $b$ are two fitting parameters. For Fig. 10(a) we find $a=1.40(0.23)$ and $b=0.041(0.13) \mathrm{mK}$. We have not yet obtained data in the low-intensity region; however, from the fit we expect temperatures near $40 \mu \mathrm{K}$ when the intensity is minimized. We also note that this result is obtained in the presence of the MOT magnetic field. Figure 10(a) demonstrates the lowest temperature for alkaline-earth atoms achieved in a single cooling step at $400 \mu \mathrm{K}$, corresponding to an rms velocity of $20 \mathrm{~cm} / \mathrm{s}$. In Fig. 10(b), for the case of $I_{t}=48 \mathrm{~mW} / \mathrm{cm}^{2}, a=2.46(0.28)$ and $b$ $=-0.31(0.20) \mathrm{mK}$. For the case of $I_{t}=27 \mathrm{~mW} / \mathrm{cm}^{2}, a$ $=1.25(0.12)$ and $b=-0.016(0.060) \mathrm{mK}$. In the highintensity case, observed temperatures are higher than the Doppler limit for detunings smaller than $40 \mathrm{MHz}$ as the Doppler cooling mechanism begins to dominate the cooling dynamics.

\section{DUAL-ISOTOPE MOT}

Dual-isotope Sr MOTs offer the possibility for sympathetically cooling relatively hot ${ }^{88} \mathrm{Sr}$ atoms with sub-Dopplercooled ${ }^{87} \mathrm{Sr}$ atoms. Slight modification of the setup shown in Fig. 2 allows simultaneous trapping of fermionic ${ }^{87} \mathrm{Sr}$ and bosonic ${ }^{88} \mathrm{Sr}$. Here the first-order diffracted light from the $80-\mathrm{MHz}$ AOM2 is used to trap ${ }^{88} \mathrm{Sr}$ while the zeroth order is used to trap ${ }^{87} \mathrm{Sr}$. To attain correct spatial overlap, the beams are combined with a beam splitter before being split into six beams. Figure 11 shows an image of the dual-isotope ${ }^{88} \mathrm{Sr}^{87} \mathrm{Sr}$ MOT under the following conditions: $\delta_{88}=-25 \mathrm{MHz}, \quad \delta_{87}=-51$ $\mathrm{MHz}$, trapping beam intensities (for both beams) of $\sim 15$ $\mathrm{mW} / \mathrm{cm}^{2}, \quad \partial B_{z}=53 \mathrm{G} / \mathrm{cm}$, and $p_{\mathrm{Sr}}=3.5 \times 10^{-8}$ Torr. For clear observation of both components in the dual MOT, we spatially separated the two isotopes by slightly misaligning the trapping beams for the ${ }^{87} \mathrm{Sr}$ MOT. A study of the number, density, and temperature of the dual-isotope MOT is currently underway.

\section{${ }^{87} \mathrm{Sr}$}
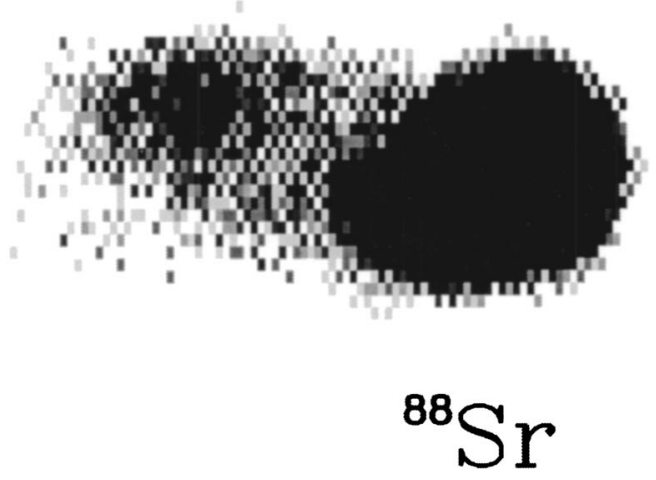

Fig. 11. Image of ${ }^{87} \mathrm{Sr}^{8}{ }^{88} \mathrm{Sr}$ fermion-boson, dual-isotope MOT. The two isotopes are deliberately separated in space for viewing clarity.

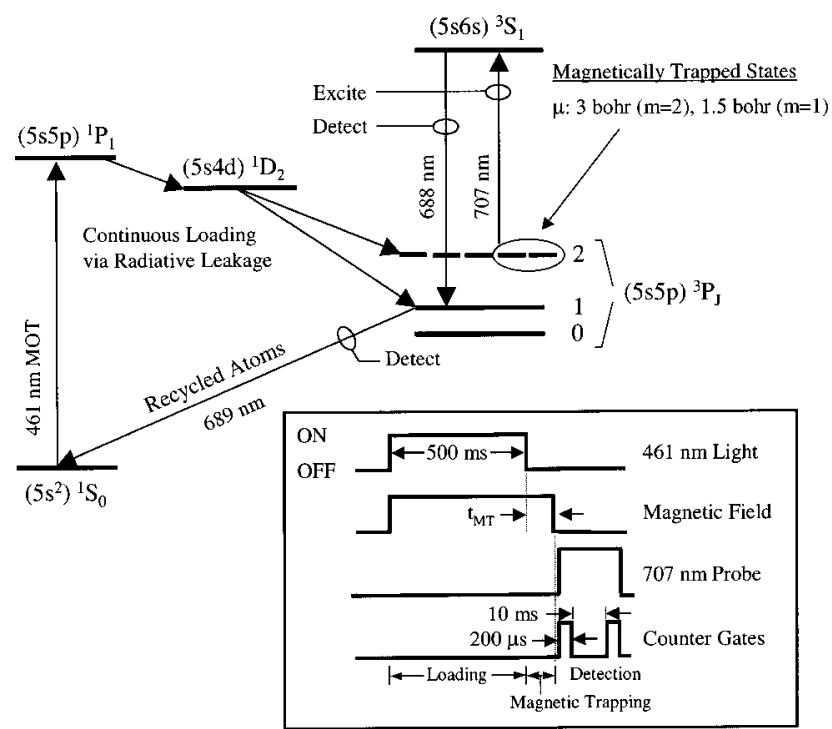

Fig. 12. Partial ${ }^{88} \mathrm{Sr}$ energy-level diagram showing transitions relevant to magnetic trapping in the ${ }^{3} P_{2}$ state. The probing scheme is also shown, with a $707-\mathrm{nm}$ probe laser and fluorescence collection at $688 \mathrm{~nm}$ and $689 \mathrm{~nm}$. The inset presents the magnetic-trap loading and detection-timing diagram.

\section{MAGNETICALLY TRAPPING ${ }^{88}$ SR IN THE ${ }^{3} \boldsymbol{P}_{2}$ METASTABLE STATE}

Since the ${ }^{1} S_{0}$ ground state lacks useful magnetic substructure, ${ }^{88} \mathrm{Sr}$ and ${ }^{87} \mathrm{Sr}$ cannot be loaded into groundstate magnetic traps currently used for alkali atoms. In the case of ${ }^{88} \mathrm{Sr}$, the ${ }^{1} S_{0}$ state completely lacks magnetic substructure, while in the case of ${ }^{87} \mathrm{Sr}$ this structure arises through coupling to the nuclear magnetic moment and thus provides, for typically employed magnetic field gradients, extremely shallow magnetic traps. As was recently pointed out, ${ }^{30,32}$ however, magnetic trapping of the alkaline-earths should be possible by using the ${ }^{3} P_{2}$ state (17-min radiative lifetime in the case of ${ }^{88} \mathrm{Sr}$ ) as an effective ground state. Moreover because of the large magnetic moment for these states [ $3 \mu_{B}$ for $\left.{ }^{3} P_{2}(m=2)\right]$ and the radiative transfer of precooled atoms from the ${ }^{1} S_{0}-{ }^{1} P_{1}$ MOT to the ${ }^{3} P_{2}$ state that exists naturally during typical MOT operations (see Fig. 12), such traps can be continuously loaded without having to produce and cool ${ }^{3} P_{2}$-state atoms separately. A similar technique has been applied to $\mathrm{Cr}$ atoms. ${ }^{31}$

Figure 12 depicts the energy levels relevant to magnetic trap loading and detection. ${ }^{32}$ The loading process consists of allowing atoms precooled in the ${ }^{1} S_{0^{-}}{ }^{1} P_{1}$ MOT to radiatively decay to the weak-field-seeking ${ }^{3} P_{2}(m$ $=1,2)$ states and then holding these atoms in the MOT quadrupole magnetic field. Zero background detection of the magnetically trapped atoms is then performed by exciting the ${ }^{3} P_{2}-{ }^{3} S_{1}$ transition $(\lambda=707 \mathrm{~nm})$ and observing the subsequent ${ }^{3} S_{1} \rightarrow{ }^{3} P_{1} \rightarrow{ }^{1} S_{0}$ radiative decay $(\lambda$ $=688$ and $689 \mathrm{~nm}$, respectively). The inset in Fig. 12 shows the specific timing used for the measurements presented here. Atoms are first loaded into the ${ }^{1} S_{0}-{ }^{1} P_{1}$ ${ }^{88} \mathrm{Sr}$ MOT for $0.5 \mathrm{~s}$. Simultaneously, ${ }^{1} P_{1}$ excited-state atoms are continuously loaded into ${ }^{3} P_{2}$ through the ${ }^{1} P_{1}$ $\rightarrow{ }^{1} D_{2} \rightarrow{ }^{3} P_{2}$ decay channel and subsequently trapped 


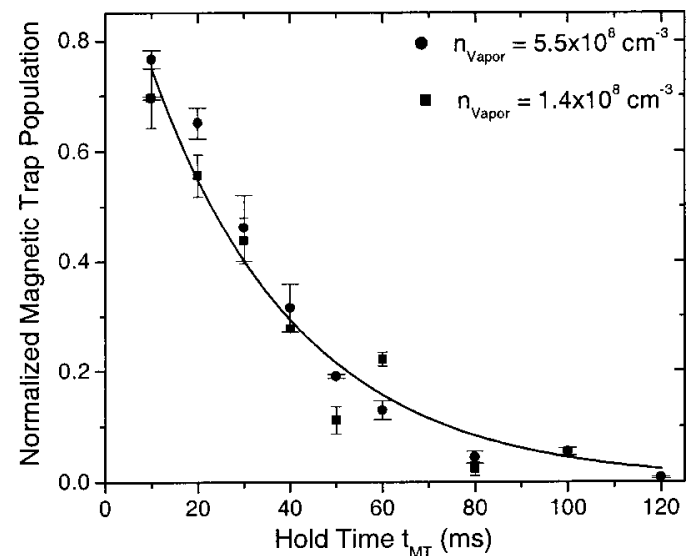

Fig. 13. Measured magnetic-trap lifetime for two different values of $\mathrm{Sr}$ vapor density.

in the weak-field-seeking ${ }^{3} P_{2}(m=2, m=1)$ states. At the end of the loading process, the 461-nm light is switched off by AOM2 within a few microseconds and atoms in the ${ }^{3} P_{2}(m=2, m=1)$ states are trapped purely by the MOT magnetic quadrupole field. After time $t_{\mathrm{MT}}$, which is the magnetic trap holding time, the magnetic field is turned off within less than $100 \mu \mathrm{s}$. A $1-\mathrm{cm}$ $\times 1-\mathrm{cm}$, on-resonance, 707-nm probe beam with an intensity of $0.22 \mathrm{~mW} / \mathrm{cm}^{2}$ illuminates the atomic cloud 100 $\mu$ s after the magnetic field is turned off. The induced fluorescence signal at 688 and $689 \mathrm{~nm}$ is then photon counted with a photomultiplier tube armed with a set of interference filters that transmit $688 \mathrm{~nm}$ and $689 \mathrm{~nm}$ and a color filter that blocks $461 \mathrm{~nm}$. To account for the lightscattering background, we use two $200-\mu$ s counter gates. The first gate is turned on $100 \mu$ s after switching off the magnetic field, and the second gate is turned on $10 \mathrm{~ms}$ later, after the trapped atoms have completely decayed away from the detection region. During each gate, the probe beam is always switched on. The hold time $t_{\mathrm{MT}}$ between probing and the ${ }^{88} \mathrm{Sr}$ MOT switch-off is then varied to map the magnetic-trap lifetime and population.

The magnetic-trap depth is expressed as $T_{\mathrm{MD}}$ $=g_{J=2} \mu_{B} m B_{m} / k_{B}$, where $g_{J=2}=1.5$ for the ${ }^{3} P_{2}$ state and $B_{m}$ is the magnitude of the maximum closed-contour of the magnetic field. Figure 13 presents magnetic-trap lifetime measurements for two different values of $p_{\mathrm{Sr}}$ and the following conditions: $T_{\mathrm{MD}}(m=2)=14 \mathrm{mK}$ and $T_{\mathrm{MD}}(m=1)=7 \mathrm{mK}$ with the magnetic field gradient of $79 \mathrm{G} / \mathrm{cm}$ and a ${ }^{1} S_{0}-{ }^{1} P_{1}$ MOT temperature of $T_{\text {MOT }}$ $=5 \mathrm{mK}$. The magnetic-trap lifetime is obtained by an exponential fit to the measured decay curves. As the $\mathrm{Sr}$ background vapor density varies from $5.5 \times 10^{8} \mathrm{~cm}^{-3}$ to $1.4 \times 10^{8} \mathrm{~cm}^{-3}$, the corresponding magnetic-trap density changes from $2.3 \times 10^{6} \mathrm{~cm}^{-3}$ to $3.1 \times 10^{5} \mathrm{~cm}^{-3}$, but the ${ }^{3} P_{2}$ trap lifetime does not change significantly; for both data sets, the lifetime is $\sim 40 \mathrm{~ms}$. Thus this unexpectedly short lifetime does not appear to depend on the Sr vapor pressure, the trapped-atom density, or collisions with $\mathrm{Sr}$ atoms from the initial MOT.

Figure 14 shows the normalized initial number of magnetically trapped atoms versus the ratio of the magnetictrap depth over the ${ }^{1} S_{0}-{ }^{1} P_{1}$ MOT temperature. This result agrees with expectations based on loading a
Maxwell-Boltzmann energy distribution into a trap of depth $k_{B} T_{\mathrm{MD}}$ [the solid curve in Fig. 14(a)]. Here the normalized initial number $N_{\text {norm }}$ of trapped atoms is given by

$$
N_{\text {norm }}=\operatorname{Erf}\left[\left(\frac{T_{\mathrm{MD}}}{T_{\mathrm{MOT}}}\right)^{1 / 2}\right]-2\left(\frac{T_{\mathrm{MD}}}{\pi T_{\mathrm{MOT}}}\right)^{1 / 2} \exp \left(-\frac{T_{\mathrm{MD}}}{T_{\mathrm{MOT}}}\right),
$$

where $\operatorname{Erf}[x]$ is the error function. However we find no clear evidence for a dependence of the magnetic-trap lifetime on the ratio of $T_{\mathrm{MD}} / T_{\mathrm{MOT}}$, as shown in Fig. 14(b). We note that the observed initial magnetic-trap population corresponds well to the measured ${ }^{1} S_{0^{-}}{ }^{1} P_{1}$ MOT population, the MOT lifetime, the magnetic-trap lifetime, and the calculated decay fraction to ${ }^{3} P_{2}(m=1,2) .{ }^{32}$

Given the relatively high vapor-cell temperature of $360{ }^{\circ} \mathrm{C}$, one must consider blackbody-radiation-induced transitions to nearby excited states as a possible source of ${ }^{3} P_{2}$ magnetic-trap loss. Here the relevant excitation rate $\left(R_{B}\right)$ is given by

$$
R_{B}=\Gamma_{1} \exp \left(-\frac{\hbar \omega_{1}}{k_{B} T_{B}}\right),
$$

where $\omega_{1}\left(\lambda_{1}\right)$ is the transition frequency (wavelength), $\Gamma_{1}$ is the Einstein $A$ coefficient, and $T_{B}$ is the blackbody temperature. In ${ }^{88} \mathrm{Sr}$, transitions from the ${ }^{3} P_{2}$ state to the $(5 s 4 d)^{3} D$ excited-state manifold $(\lambda \sim 3 \mu \mathrm{m})$ are especially relevant to magnetic-trap loss. In particular, Fig. 15 shows three possibilities wherein ${ }^{3} P_{2}-4 d^{3} D_{J}$ blackbody-induced absorption processes depopulate the magnetic trap. In Fig. 15(a) excitation of the
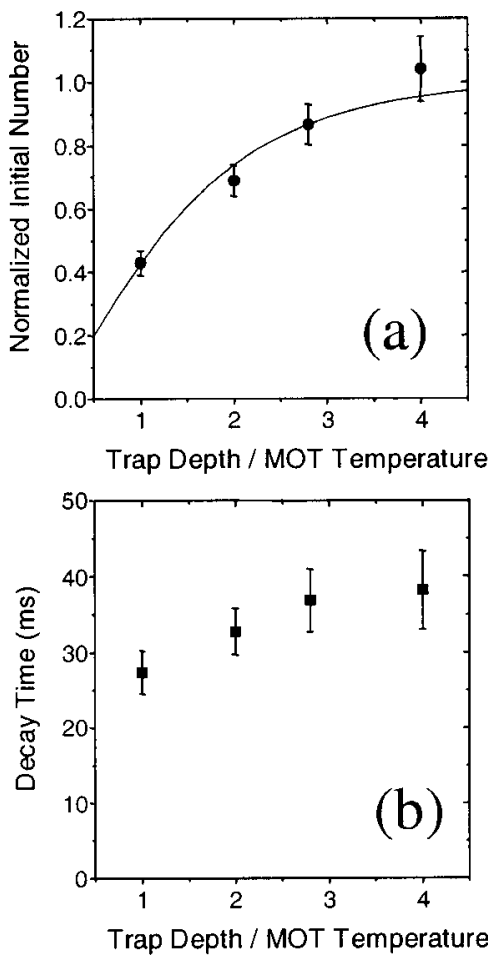

Fig. 14. Magnetic trap (a) number and (b) lifetime versus the ratio of trap depth over sample temperature. The solid curve in (a) is a theoretical prediction based on loading a MaxwellBoltzmann energy distribution into a magnetic trap of depth $k_{B} T_{\mathrm{MD}}$. 
(a)

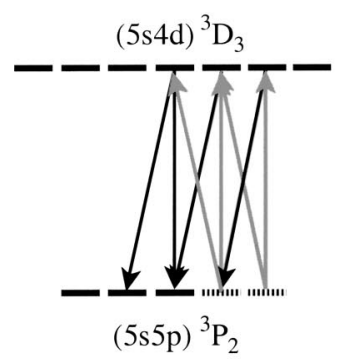

(b)

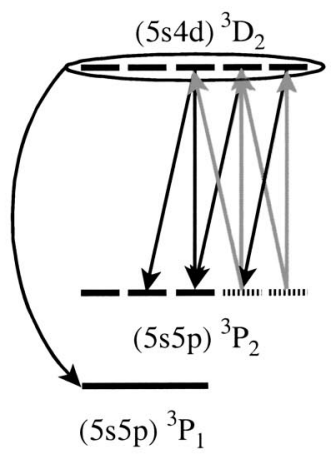

(c)

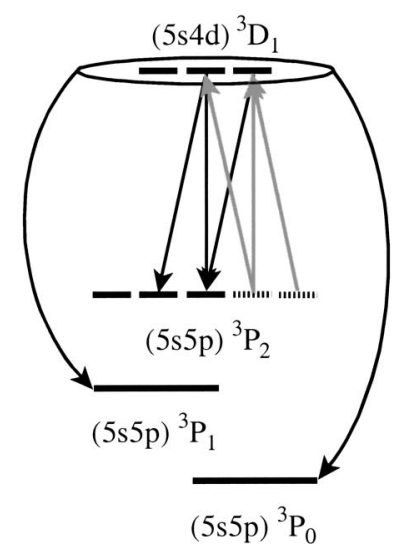

Fig. 15. Blackbody radiation processes that induce magnetictrap loss. In (a) excitation of the ${ }^{3} P_{2}-4 d^{3} D_{3}$ transition depolarizes the magnetically trapped gas while in (b) and (c), excitation of the ${ }^{3} P_{2}-4 d^{3} D_{2}$ and ${ }^{3} P_{2}-4 d^{3} D_{1}$ transitions depolarizes the gas and permits transitions to untrapped fine-structure states. Magnetically trapped ${ }^{3} P_{2}$ substates are shown as dotted lines while absorption (spontaneous emission) events that induce trap loss are shown as gray (black) arrows.

${ }^{3} P_{2}-4 d^{3} D_{3} \quad$ transition $^{42} \quad\left(\lambda_{1}=2.923 \mu \mathrm{m}, \Gamma_{1}=1.4\right.$ $\times 10^{6} \mathrm{~s}^{-1}$ ) depolarizes the magnetically trapped gas, while in Figs. 15(b) and 15(c) excitation of the ${ }^{3} P_{2}-4 d^{3} D_{2}$ and ${ }^{3} P_{2}-4 d^{3} D_{1}$ transitions $\quad\left(\lambda_{1}\right.$ $=3.012 \mu \mathrm{m}, \quad \Gamma_{1}=3.6 \times 10^{5} \mathrm{~s}^{-1}$ and $\lambda_{1}=3.067 \mu \mathrm{m}$, $\Gamma_{1}=4 \times 10^{4} \mathrm{~s}^{-1}$, respectively) depolarize the gas and permit decay to untrapped fine-structure states.

As a first estimate of the time scale for these trap-loss processes, we assume a blackbody temperature of $360^{\circ} \mathrm{C}$ and an extreme case in which single ${ }^{3} P_{2}-4 d^{3} D_{J}$ absorption events remove a ${ }^{3} P_{2}$ atom from the trap. Summing over the individual rates given by Eq. (4) then gives a predicted $1 / e$ trap lifetime of only $1.2 \mathrm{~ms}$. Using a more realistic blackbody temperature of $190^{\circ} \mathrm{C}$, estimated by accounting for the solid angle subtended by the cell windows, gives a predicted $1 / e$ trap lifetime of $21 \mathrm{~ms}$, in rough agreement with the observed lifetime of $\sim 35 \mathrm{~ms}$. Clearly, blackbody-induced transitions dominate the observed trap loss. Obtaining longer lifetimes thus requires performing the magnetic trapping experiments in a room-temperature apparatus, such as a beam-loaded $\mathrm{Sr}$ trap. Here the lifetime resulting solely from blackbody radiation should exceed $10 \mathrm{~s}$, sufficiently long to perform useful experiments.

\section{CONCLUSIONS}

We have investigated the dynamics of the ${ }^{88} \mathrm{Sr}$ MOT in detail, and have found that the cooling rates agree well with Doppler cooling theory, while the heating rate is higher than theoretical predictions. We find that an optimal intensity and detuning can maximize the trapped-atom density in a Sr MOT, and the lifetime and number of trapped atoms in the ${ }^{88} \mathrm{Sr}$ MOT can be increased by a factor of 10 by employing two repumping lasers. In the ${ }^{87} \mathrm{Sr}$ MOT, we have also observed the lowest temperature for alkaline-earth atoms using a single cooling stage in which polarization-gradient cooling is effected by the presence of a nuclear-spin-originated magnetic degeneracy in the ground state. We will continue to explore the prospect of a high-quality, optical-frequency standard based on cold ${ }^{87} \mathrm{Sr}$ atoms. In addition, by magnetically trapping ${ }^{88} \mathrm{Sr}$ in the metastable ${ }^{3} P_{2}$ state, we have made the first magnetic trap for alkaline-earth atoms. The surprisingly short trap lifetime is attributed to blackbody-radiationinduced transitions. In future beam-loaded trap experiments, sub-Doppler cooling based on the radiatively closed $(5 s 4 d)^{3} D_{3}-(5 s 5 p)^{3} P_{2}$ transition can be applied, followed by radio-frequency evaporative cooling. One target is quantum degeneracy in the ${ }^{3} P_{2}$ metastable state. Another target is the production of an ultracold sample in a lattice-bound Lamb-Dicke regime for the development of an optical-frequency standard. We plan further polarization-gradient cooling measurements in the ${ }^{87} \mathrm{Sr}$ MOT through use of the intercombination, narrow-line transition, investigating various approaches to the quantum degeneracy. Finally we will study cold collisions using ultracold and dense $\mathrm{Sr}$ atoms in both the ground and metastable ${ }^{3} P$ states.

\section{ACKNOWLEDGMENTS}

We gratefully acknowledge stimulating discussions with U. Sterr, C. Oates, and T. Ido. This work is funded by the National Science Foundation, the Office of Naval Research, and the National Institute of Standards and Technology. T. H. Loftus appreciates support from the National Research Council.

Jun Ye may be reached by e-mail at ye @jila.colorado.edu.

\section{REFERENCES}

1. T. W. Hänsch and A. L. Schawlow, "Cooling of gases by laser radiation,” Opt. Commun. 13, 68-69 (1975).

2. J. P. Gordon and A. Ashkin, "Motion of atoms in a radiation trap," Phys. Rev. A 21, 1606-1617 (1980).

3. S. Stenholm, "The semiclassical theory of laser cooling," Rev. Mod. Phys. 58, 699-739 (1986).

4. P. D. Lett, W. D. Phillips, S. L. Rolston, C. E. Tanner, R. N. Watts, and C. I. Westbrook, “Optical molasses," J. Opt. Soc. Am. B 6, 2084-2107 (1989).

5. E. L. Raab, M. Prentiss, A. Cable, S. Chu, and D. E. Pritchard, "Trapping of neutral sodium atoms with radiation pressure,” Phys. Rev. Lett. 59, 2631-2634 (1987). 
6. J. Dalibard and C. Cohen-Tannoudji, "Laser cooling below the Doppler limit by polarization gradients: simple theoretical models," J. Opt. Soc. Am. B 6, 2023-2045 (1989).

7. D. S. Weiss, E. Riis, Y. Shevy, P. J. Ungar, and S. Chu, "Optical molasses and multilevel atoms: experiment," J. Opt. Soc. Am. B 6, 2072-2083 (1989).

8. C. Monroe, W. Swann, H. Robinson, and C. Wieman, "Very cold trapped atoms in a vapor cell," Phys. Rev. Lett. 65, 1571-1574 (1990).

9. K. Lindquist, M. Stephens, and C. Wieman, "Experimental and theoretical study of the vapor-cell Zeeman optical trap," Phys. Rev. A 46, 4082-4090 (1992).

10. C. Salomon, J. Dalibard, W. D. Phillips, A. Clairon, and S. Guellati, "Laser cooling of cesium atoms below $3 \mu \mathrm{K}$," Europhys. Lett. 12, 683-699 (1990).

11. S. Chang, T. Y. Kwon, H. S. Lee, and V. G. Minogin, "Twophoton laser-cooling mechanism in multilevel interaction schemes," Phys. Rev. A 60, 3148-3159 (1999).

12. H. Katori, T. Ido, Y. Isoya, and M. Kuwata-Gonokami, "Magneto-optical trapping and cooling of strontium atoms down to the photon recoil temperature," Phys. Rev. Lett. 82, 1116-1119 (1999).

13. K. R. Vogel, T. P. Dinneen, A. Gallagher, and J. L. Hall, "Narrow-line Doppler cooling of strontium to the recoil limit," IEEE Trans. Instrum. Meas. 48, 618-621 (1999).

14. T. Ido, Y. Isoya, and H. Katori, "Optical-dipole trapping of Sr atoms at a high phase-space density," Phys. Rev. A 61, 061403 (2000).

15. T. Kuwamoto, K. Honda, Y. Takahashi, and T. Yabuzaki, "Magneto-optical trapping of $\mathrm{Yb}$ atoms using an intercombination transition," Phys. Rev. A 60, (R)745-748 (1999).

16. E. A. Curtis, C. W. Oates, and L. Hollberg, "Quenched narrow-line laser cooling of ${ }^{40} \mathrm{Ca}$ to near the photon recoil limit," Phys. Rev. A 64, 031403 (2001).

17. T. Binnewies, G. Wilpers, U. Sterr, F. Riehle, J. Helmcke, T. E. Mehlstaubler, E. M. Rasel, and W. Ertmer, "Doppler cooling and trapping on forbidden transitions," Phys. Rev. Lett. 87, 123002 (2001).

18. J. Grünert and A. Hemmerich, "Sub-Doppler magnetooptical trap for calcium," Phys. Rev. A 65, 041401 (2002).

19. Y. Bidel, B. Klappauf, J. C. Bernard, D. Delande, G. Labeyrie, C. Miniature, D. Wilkowski, and R. Kaiser, "Coherent light transport in a cold strontium cloud," Phys. Rev. Lett. 88, 203902 (2002)

20. X. Y. Xu, T. H. Loftus, M. J. Smith, J. L. Hall, A. Gallagher, and J. Ye, "Dynamics in a two-level atom magneto-optical trap," Phys. Rev. A 66, 011401 (2002).

21. F. Ruschewitz, J. L. Peng, H. Hinderthur, N. Schaffrath, K H. Sengstock, and W. Ertmer, "Sub-kilohertz optical spectroscopy with a time domain atom interferometer," Phys. Rev. Lett. 80, 3173-3176 (1998).

22. F. Riehle, H. Schnatz, G. Zinner, K. Zeiske, B. Lipphardt and J. Helmcke, "Calcium optical frequency standard based on atom interferometry," Laser Phys. 6, 237-243 (1996).

23. F. Riehle, H. Schnatz, B. Lipphardt, G. Zinner, T. Trebst, and J. Helmcke, "The optical calcium frequency standard," IEEE Trans. Instrum. Meas. 48, 613-617 (1999),

24. T. Kisters, K. Zeiske, F. Riehle, and J. Helmcke, "High- resolution spectroscopy with laser-cooled and trapped calcium atoms," Appl. Phys. B 59, 89-98 (1994).

25. T. Udem, S. A. Diddams, K. R. Vogel, C. W. Oates, E. A. Curtis, W. D. Lee, W. M. Itano, R. E. Drullinger, J. C. Bergquist, and L. Hollberg, "Absolute frequency measurements of the $\mathrm{Hg}^{+}$and Ca optical clock transitions with a femtosecond laser," Phys. Rev. Lett. 86, 4996-4999 (2001).

26. J. Weiner, V. Bagnato, S. Zilio, and P. S. Julienne, "Experiments and theory in cold and ultracold collisions," Rev. Mod. Phys. 71, 1-85 (1999).

27. T. P. Dinneen, K. R. Vogel, E. Arimondo, J. L. Hall, and A. Gallagher, "Cold collision of $\mathrm{Sr}^{*}$-Sr in a magneto-optical trap," Phys. Rev. A 59, 1216-1222 (1999).

28. G. Zinner, T. Binnewies, F. Riehle, and E. Tiemann, "Photoassociation of cold Ca atoms," Phys. Rev. Lett. 85, 2292 2295 (2000).

29. H. Katori, "Spectroscopy of strontium atoms in the LambDicke confinement," in Proceedings of the 6th Symposium on Frequency Standards and Metrology, P. Gill, ed. (World Scientific, Singapore, 2002), pp. 323-330.

30. A. Derevianko, "Feasibility of cooling and trapping metastable alkaline-earth atoms," Phys. Rev. Lett. 87, 023002 (2001)

31. J. Stuhler, P. O. Schmidt, S. Hensler, J. Werner, J. Mlynek, and T. Pfau, "Continuous loading of a magnetic trap," Phys. Rev. A 64, 031405 (2001)

32. T. Loftus, J. R. Bochinski, and T. W. Mossberg, "Magnetic trapping of ytterbium and the alkaline earth metals," Phys. Rev. A 66, 013411 (2002)

33. J. Goldwin, S. B. Papp, B. DeMarco, and D. S. Jin, "Twospecies magneto-optical trap with ${ }^{40} \mathrm{~K}$ and ${ }^{87} \mathrm{Rb}$," Phys. Rev. A 65, 021402 (2002).

34. M.-O. Mewes, G. Ferrari, F. Schreck, A. Sinatra, and C. Salomon, "Simultaneous magneto-optical trapping of two lithium isotopes," Phys. Rev. A 61, 011403 (1999).

35. S. G. Crane, X. Zhao, W. Taylor, and D. J. Vieira, "Trapping an isotopic mixture of fermionic ${ }^{84} \mathrm{Rb}$ and bosonic ${ }^{87} \mathrm{Rb}$ atoms," Phys. Rev. A 62, 011402 (1999).

36. T. Loftus, J. R. Bochinski, and T. W. Mossberg, "Simultaneous multi-isotope trapping of ytterbium," Phys. Rev. A 63 053401 (2001)

37. T. Kurosu and F. Shimizu, "Laser cooling and trapping of calcium and strontium,” Jpn. J. Appl. Phys., 29, L2127L2129 (1990)

38. T. Kurosu and F. Shimizu, "Laser cooling and trapping of alkaline earth atoms," Jpn. J. Appl. Phys. 31, 908-912 (1992)

39. L. R. Hunter, W. A. Walker, and D. S. Weiss, "Observation of an atomic Stark-electric quadrupole interference," Phys. Rev. Lett. 56, 823-826 (1986).

40. C. W. Bauschlicher, S. R. Langhoff, and H. Partridge, "The radiative lifetime of the ${ }^{1} D_{2}$ state of $\mathrm{Ca}$ and $\mathrm{Sr}$ : a corevalence treatment," J. Phys. B: At. Mol. Phys. 18, 15231532 (1985).

41. K. Vogel, Ph.D. thesis (University of Colorado, Boulder, 1999).

42. H. G. C. Werij, C. H. Greene, C. E. Theodosiou, and A. Gallagher, "Oscillator strengths and radiative branching ratios in atomic Sr," Phys. Rev. A 46, 1248-1260 (1992). 